with the low concentrations of human apotransferrin and ceruloplasmin in premature infants found by other workers. ${ }^{23}$

The possible antioxidant role of plasma dl$\alpha$-tocopherol in neonatal disorders such as retinopathy of prematurity has received much attention. ${ }^{5}$ The present findings raise the possibility that deficiencies in the potent human apotransferrin/ ceruloplasmin dependent antioxidant system may be important in the pathogenesis of retinopathy of prematurity and similar disorders.

These findings are relevant to the suggestion ${ }^{6}$ that increased plasma concentrations of non-haem iron in the neonatal period are of central importance in the pathogenesis of retinopathy of prematurity. Large increases in serum ferritin and transferrin iron saturation are usually seen in premature infants and infants born at full term shortly after birth. Additional sources of exogenous iron in the neonatal period include dietary iron supplementation and blood transfusion. ${ }^{6}$ The present data suggest that one important effect of increased non-haem iron may be to neutralise much of the premature infant's scarce antioxidant activity, thereby sharply increasing vulnerability to oxygen.

We thank Dr DL Phelps and Dr WA Silverman for their helpful suggestions, Dr DM Purohit for help in obtaining samples of cord serum, and American Red Cross Blood Services, Carolina Low Country Region for samples of serum from blood donors. RBN had a postdoctoral fellowship from the College of Graduate Studies of the Medical University of South Carolina, and the work was supported by the Veterans Administration.

\section{References}

' Stocks J, Gutteridge JMC, Sharp RJ, Dormandy TL. The inhibition of lipid autoxidation by human serum and its relation to serum proteins and $\alpha$-tocopherol. Clinical Science and Molecular Medicine 1974:47:223-33.

2 Hilderbrand DC, Fahim Z, James E, Fahim M. Ceruloplasmin and alkaline phosphatase levels in cord serum of term, preterm and physiologically jaundiced neonates. Am J Obstet Gynecol 1974:118:950-4.

${ }^{3}$ Scott PH, Berger HM, Kenward C, Scott P, Wharton BA. Effect of gestational age and intrauterine nutrition on plasma transferrin and iron in the newborn. Arch Dis Child 1975;50: 796-8.

+ Gutteridge JMC, Stocks J. Caeruloplasmin: physiological and pathological perspectives. CRC Crit Rev Clin Lab Sci 1981;14: 257-329.

5 Phelps DL, Rosenbaum AL, Isenberg SJ, Leake RD, Dorey FJ. Tocopherol efficacy and safety for preventing retinopathy of prematurity: a randomized, controlled, double-masked trial. Pediatrics 1987;79:489-500.

6 Sullivan JL. Retinopathy of prematurity and iron: a modification of the oxygen hypothesis. Pediatrics 1986;78:1171-2.

Correspondence and requests for reprints to Dr JL Sullivan. VAMC (113), 109 Bee Street, Charleston. SC 29403, USA.

Accepted 18 December 1987

\title{
Simple method for securing umbilical catheters
}

\author{
M SOUTH* AND A MAGNAY†
}

${ }^{*}$ Department of Paediatrics, University of Cambridge, and †Birmingham Maternity Hospital

SUMMARY A method for securing umbilical catheters is described. It has been used successfully in over 350 babies. It is a simple and rapid method that allows for easy repositioning and, unlike some established techniques, has no potential for damage to the preterm baby's skin.

Since their introduction in the early 1960s the techniques of umbilical arterial and venous catheterisation have been accepted into routine clinical practice. Adequate fixation of catheters is important: displacement can lead to malposition of the catheter tip, with consequent poor sampling and increased risk of vascular thrombosis; catastrophic haemorrhage can occur if the catheter 'falls out.'

Most recommended methods for catheter fixation involve a combination of typing loops of suture material around the base of the catheter, and the use of a 'goalpost' bridge of adhesive tape attached to the catheter and to the skin of the abdominal wall. ${ }^{1}$ Having used such methods for a number of years we became aware of several problems: tying the loops of suture material is fiddly, and it can be difficult to tie them sufficiently tight to grip the catheter without partially occluding its lumen. The adhesive tape is often difficult to attach to the baby's moist, vernix covered skin. The tape reduces the area available for the use of transcutaneous electrodes. The bridge of tape can be caught accidentally during handling, and it has even provided an effective footstrap for an active baby to kick against. Removal of adhesive tape from the often extremely fragile skin of a very preterm baby can be damaging and can lead to ooze of plasma, increased fluid loss, and risk of sepsis. Once fixation is complete it can be difficult to reposition the catheter if a radiograph shows an unsatisfactory placement of the tip. 

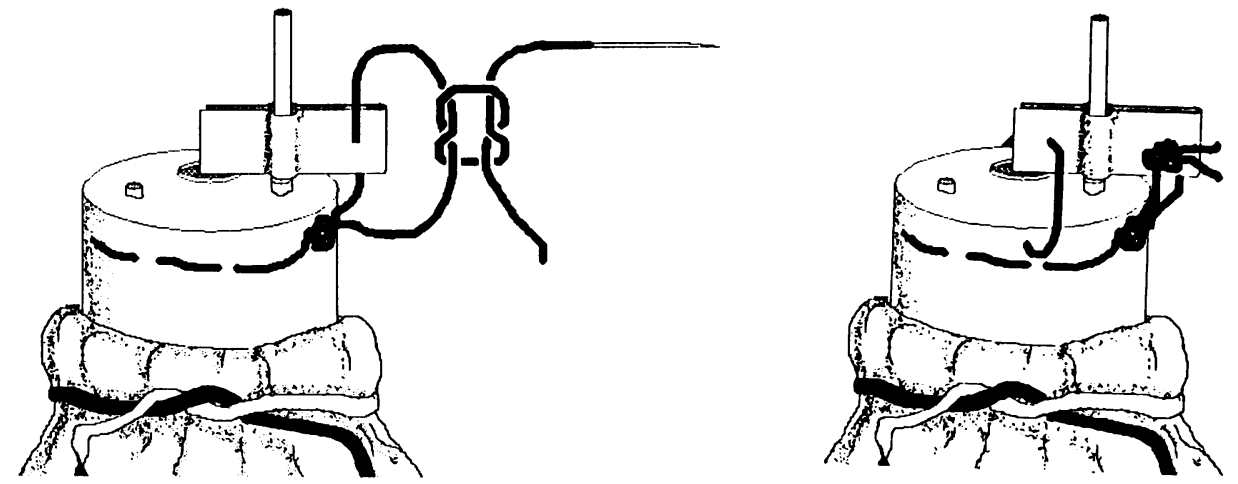

Figure Left-purse-string suture is passed through the zinc oxide tape and tied. Right-second suture is passed through the cord stump to transfix the umbilical vein. It is then taken through the zinc oxide tape on the opposite side to the first suture and then tied.

In an attempt to overcome some of these problems we have used a different method of fixation.

\section{Method}

When the catheter had been inserted to the required depth, a purse-string $3 / 0$ black silk suture was placed into the cord stump, the needle was left attached to one free end. The base of the catheter was dried with a gauze swab and a piece of $1 \mathrm{~cm}$ wide zinc oxide tape (no other tape will do) about $2 \mathrm{~cm}$ long was folded around the catheter as close as possible to the cord stump. The needle of the suture was then passed through the tape close to the catheter and tied (fig-left). The needle, with a long thread attached, was then cut off close to the knot. The needle was passed through the cord stump to transfix the umbilical vein, through the zinc oxide tape on the other side of the catheter, and then tied (fig-right). No further fixation was used.

We preferred to place the tip of umbilical arterial catheters at the level of the diaphragm. If assessment by radiography showed the catheter position to be unsatisfactory, it was withdrawn either to the diaphragmatic level (for catheters placed too high) or the low (L3-4) position (for catheters placed too low). To achieve this the zinc oxide tape was cut on either side, close to the base of the catheter, leaving the two free pieces of tape attached by the sutures to the cord stump. The catheter was then withdrawn the measured distance and a fresh piece of zinc oxide tape was folded around the base of the catheter incorporating the two pieces of tape still attached to the stump.

\section{Results}

Since February 1985 more than 350 umbilical catheters have been secured using the method described. Three catheters became accidentally dislodged. The babies were over 2 weeks of age and were out of their incubators being weighed at the time. The fixation sutures and zinc oxide tape were all still firmly attached to the catheter, but the dried up cord had separated from the cutaneous stump leaving no secure fixation.

\section{Discussion}

This method of catheter fixation was found to be simpler and more rapid to apply than other methods. It does not lead to damage to the skin and allows for easy repositioning. The method is similar to that briefly described by Adams and Rudolph ${ }^{2}$ but in our method the tape is stitched to the cord stump and we do not use any form of tape around the infant's waist.

No records were kept when previous methods were in use, however, the rate of catheter displacement has certainly fallen since the new technique was introduced. Three displacements occurred with catheters that were still in place after the normal time at which the cord separates. Although most catheters are removed before this time, we are attempting to prevent the problem by placing one of the sutures through the cutaneous part of the cord stump.

\section{References}

1 Klauss MH, Fanaroff AA, eds. Care of the high risk neonate. 3rd ed. Philadelphia: WB Saunders, 1986:431.

2 Adams JM, Rudolph AJ. Simple method for stabilising umbilical catheters. Arch Dis Child 1979;54:563.

Correspondence to Dr M South, 69 Royal Parade, Parkville, Victoria 3052, Australia.

Accepted 5 November 1987 